\title{
An IoT Knowledge Reengineering Framework for Semantic Knowledge Analytics for BI-Services
}

\author{
Nilamadhab Mishra, Hsien-Tsung Chang, and Chung-Chih Lin \\ Department of Computer Science and Information Engineering, Chang Gung University, 259 Wen-Hwa 1st Road, \\ Kwei-Shan, Tao-Yuan 333, Taiwan \\ Correspondence should be addressed to Chung-Chih Lin; cclin@mail.cgu.edu.tw
}

Received 10 February 2015; Revised 19 May 2015; Accepted 21 May 2015

Academic Editor: Sanghyuk Lee

Copyright (C) 2015 Nilamadhab Mishra et al. This is an open access article distributed under the Creative Commons Attribution License, which permits unrestricted use, distribution, and reproduction in any medium, provided the original work is properly cited.

\begin{abstract}
In a progressive business intelligence (BI) environment, IoT knowledge analytics are becoming an increasingly challenging problem because of rapid changes of knowledge context scenarios along with increasing data production scales with business requirements that ultimately transform a working knowledge base into a superseded state. Such a superseded knowledge base lacks adequate knowledge context scenarios, and the semantics, rules, frames, and ontology contents may not meet the latest requirements of contemporary BI-services. Thus, reengineering a superseded knowledge base into a renovated knowledge base system can yield greater business value and is more cost effective and feasible than standardising a new system for the same purpose. Thus, in this work, we propose an IoT knowledge reengineering framework (IKR framework) for implementation in a neurofuzzy system to build, organise, and reuse knowledge to provide BI-services to the things (man, machines, places, and processes) involved in business through the network of IoT objects. The analysis and discussion show that the IKR framework can be well suited to creating improved anticipation in IoT-driven BI-applications.
\end{abstract}

\section{Introduction}

Business intelligence services (BI-services) must take advantage of the rapid evolution of the IoT (Internet of Things) network to connect millions of IoT objects that are active in the contemporary business environment. The advancement of IoT applications is advantageous to numerous BIapplications such as tourism, healthcare, manufacturing, transportation and logistics management, and supply chain management [1]. The IoT enables a network of smart objects, such as sensors, RFIDs (radio frequency identifiers), and other sensing and computing technologies in the BIenvironment, to ensure globally distributed data delivery to cloud applications, from which the bulk of IoT data can be accessed for purposes of business analytics without any network or communication barriers. The modernisation of IoT and automation requires enhanced connectivity, such as any-place, any-process, any-people, any-thing, and anytime connectivity among networked IoT objects, and forms a comprehensive IoT environment that can be used to regulate numerous BI-applications. A BI-application in a cloud-based IoT environment functions as a business performance monitoring service through semantic knowledge analytics of multidimensional data that are aggregated from the distributed business environment $[2,3]$. In a large-scale IoT environment, large amounts of semistructured and unstructured data are produced in real time and present difficulties to IoT knowledge analytics when searching for needed values from the data. The structured and unstructured data generated from individual IoT sources can be seamlessly integrated and analysed through an ontology model embedded with a semantic knowledge analytics mechanism [4]. Several problems, such as managing heterogeneous knowledge, transforming data into knowledge, transforming knowledge into actions, transforming actions into cognitive intelligence, and tuning the IoT knowledge to regulate the BI-applications, are faced in a business IoT environment. In any real-time BIapplication, the IoT knowledge base creates awareness about what knowledge may be used to obtain a better trade value, whereas the cognitive intelligence acts as a recommender 


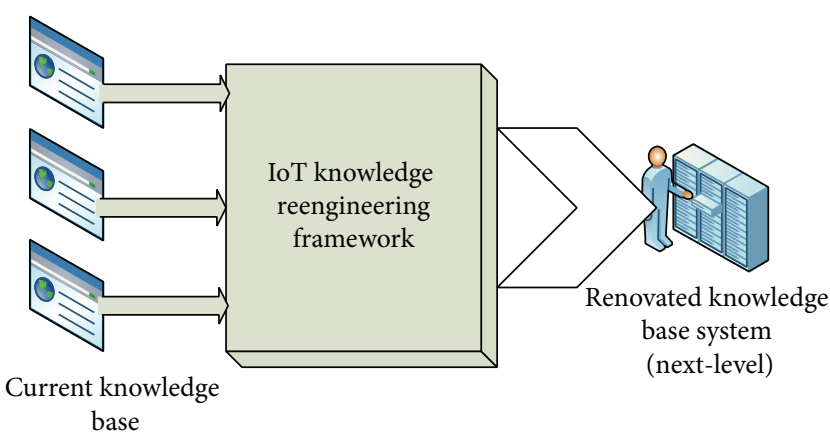

FIGURE 1: Mapping outline between the knowledge bases.

to suggest when and how to use the knowledge to obtain a better trade value $[5,6]$. Thus, both IoT knowledge bases and cognitive intelligence perform major roles in modernising the BI-environment into a real cognitive state to provide wide-ranging BI-services through semantic knowledge interpretation and recommendation [7]. However, rapidly changing knowledge context scenarios, such as semantics, rules, frames, and ontology along with data scale, structure, and level of abstraction, require rapid reengineering efforts and force the current running knowledge base into a superseded state [8].

Thus, to understand the breach between the current superseded knowledge base and the renovated knowledge base system, a mapping outline is sketched in Figure 1. The IoT knowledge reengineering framework transforms the current superseded knowledge base into a renovated knowledge base system for effective use in BI-services.

In this work, we propose an IKR framework for the effective transformation of a current superseded IoT knowledge base into a renovated knowledge base system that can yield better business value and be more cost effective than standardising a new system for the same purpose. The IKR framework can build, organise, and reuse the knowledge from the current superseded IoT knowledge base to provide BIservices aimed at self-regulated decisions, actuations, control, and coordinations of the things (man, machine, and process) involved in BI-services through networked IoT objects $[9,10]$.

To perform the knowledge analytic and reanalytic operations in the IKR framework, we suggest an implementation through a neurofuzzy system. We propose four algorithms to perform various knowledge analytic operations, the first three of which are the knowledge reanalytics operation, reverse knowledge analytic operation, and forward knowledge analytic operation. Further, we propose an implementation algorithm for neurofuzzy analytics that can be used to produce knowledge inferences with higher probabilities. In our empirical environment, we use a gradient algorithm as a learning algorithm for the integrated neurofuzzy system. The fuzzy system has a better knowledge representation ability compared to the neural system, and the neural system has better learning and knowledge inference abilities compared to the fuzzy system. Thus, the hybridisation of the neural system and fuzzy system (neurofuzzy system) provides a better knowledge representation as well as learning and knowledge inference abilities.

The remainder of this paper is organised as follows. Section 2 discusses the IKR framework organisations towards building the renovated knowledge base system through knowledge engineering and reengineering agendas that can be applied to different BI-services. Section 3 highlights the analysis and discussion of the proposed framework along with the prospective review, analysis, and system implementations. Finally, Section 4 concludes this paper and suggests future work.

\section{IKR Framework Organisation}

Several studies have applied knowledge engineering and reengineering systems, frameworks, tools, and other aspects to various BI-services. However, we propose an IKR framework that addresses the growing amount of business knowledge through a redevelopment strategy. The IKR framework organisation typically uses a reengineering framework to transform a current superseded IoT knowledge base into an active renovated knowledge base system; the detailed framework architecture is described in Figure 2. In the reengineering framework, we suggest a merging of the current knowledge contexts, rules, and ontologies with the ever-expanding business knowledge to formulate the backbone of a renovated knowledge base system. Current knowledge contexts, rules, and ontologies can provide only the semantic knowledge analytic scenarios of previous BI-services, problem findings, domain knowledge, technical solutions, risk anticipation and analytics, strategy formulation and anticipation, and so forth.

A current IoT knowledge base can be characterised as a superseded knowledge base that lacks adequate knowledge context scenarios as well as semantics, rules, frames, and ontology contents and thus may not meet the latest requirements of contemporary BI-services. A current IoT knowledge base can be analysed to extract adequate knowledge context scenarios, and the next-level IoT knowledge base can be analysed to extract the new knowledge context scenarios. The integration of current knowledge context scenarios with next-level knowledge context scenarios produces an explicit knowledge finding and integrated knowledge base that can effectively meet BI-service requirements.

The new knowledge components can be built from the integrated knowledge base and ensure continued building on the new knowledge base system. To visualise the overall IKR organisation framework, a stepwise analysis is performed.

Step 1. Analyse the current IoT knowledge base.

Step 2. Perform knowledge extraction to acquire the current knowledge context scenarios.

Step 3. Set and configure a new IoT database, from which the new knowledge context scenarios can be extracted.

Step 4. Perform explicit knowledge findings and integrations through the current knowledge context scenarios and the next-level knowledge context scenarios. 


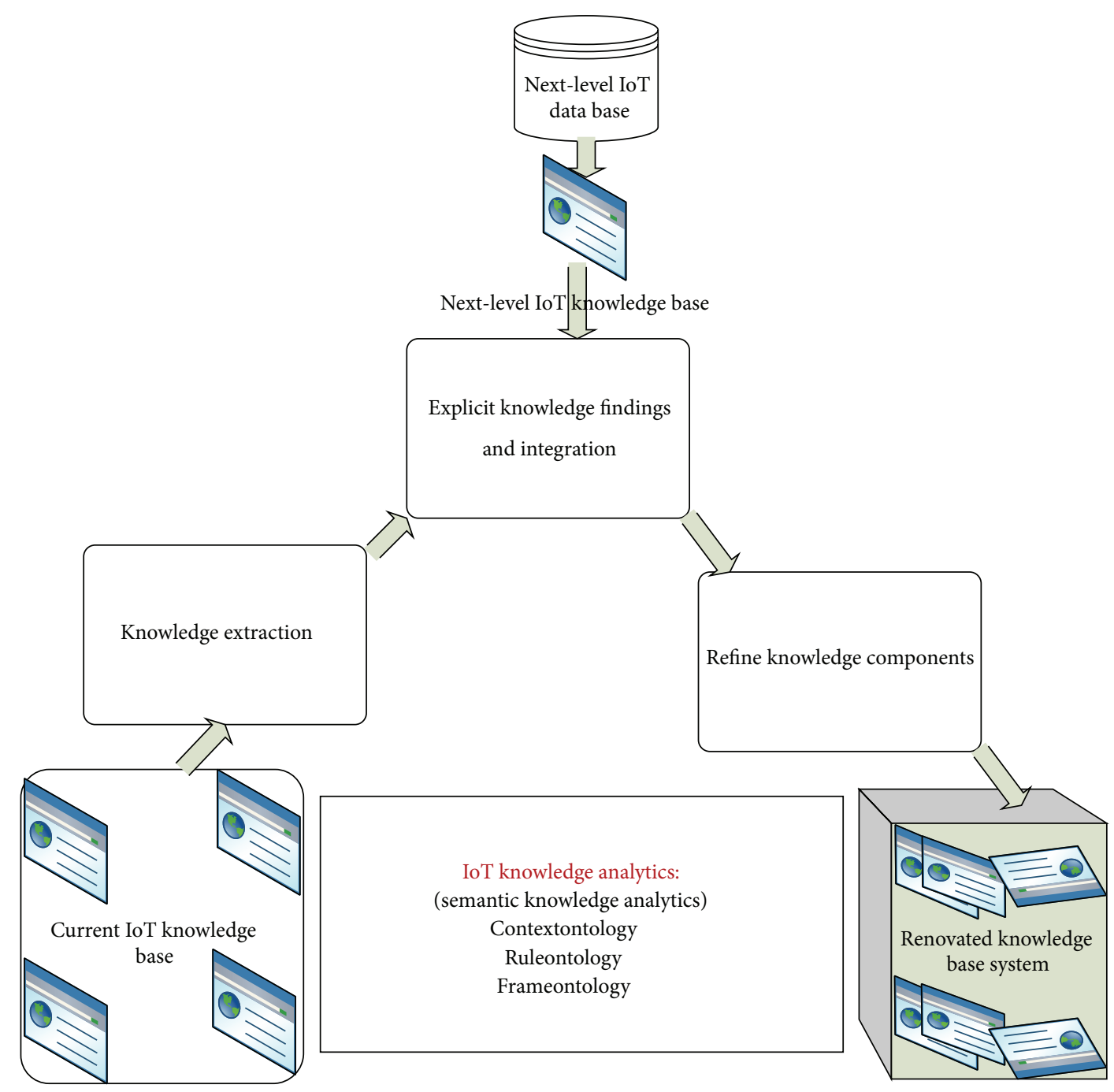

FIGURE 2: IKR framework organisation.

Step 5. Build new knowledge components, such as new semantics, rules, frames, and ontologies that are well suited to current BI settings.

Step 6. Finally, reconstruct the renovated knowledge base system.

In the IKR framework, reverse analytics are integrated with forward analytics to create a comprehensive reengineering framework. Reverse analytics are substantially more useful to study for determining the consequences of current $\mathrm{BI}$-decisions and actions in addition to the analytics of risks that are anticipated in the business. The knowledge residing in the mind of a business expert that has not been documented anywhere may be treated as tactical business knowledge. Tactical business knowledge can be transformed into an explicit knowledge base through standard documentation by ensuring ease of understanding and use. The explicit knowledge can be effectively used in BI-services towards monitoring business activities and functions that are coordinated within the frame of the IoT environment.

Figure 3 describes the possible steps in IoT knowledge analytic operations that can be used to effectively reuse the current superseded IoT knowledge base system. In a current IoT knowledge base, the following operational steps may be taken to obtain an enriched next-level IoT knowledge base that can effectively meet BI-service requirements.

The IoT knowledge analytic operations should be automated to perform the dynamic enhancement of the knowledge base per the current BI consequences.

Step 1. Analyse knowledge dimensions and supported functions-knowledge dimensions refer to the features of knowledge context scenarios that should be initially reformatted per the current architecture of the BI-services. In an IoT knowledge base system, attribute renaming and rescaling are also included in reformatting the knowledge dimensions. Here, a mapping may be performed between the 


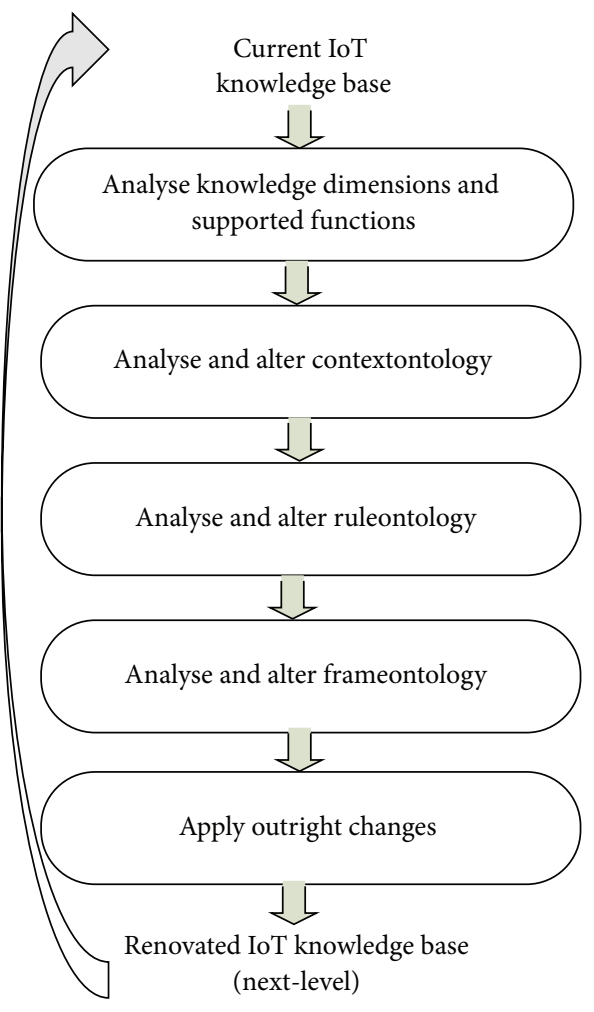

FIgURE 3: IoT knowledge analytic cycle.

functions of the knowledge base system and the BI-services to identify and resolve gaps and obstacles.

Step 2. Analyse and alter context ontology-this step defines the ontology mechanism based on information contexts. Here, based on the BI-services, the addition and deletion of knowledge contexts can be performed to ensure that the knowledge base remains current and useful.

Step 3. Analyse and alter rule ontology-this step defines the ontology mechanism based on complex fuzzy rules. Based on the requirements of the BI-services, specific knowledge components, such as semantic rules, can be dynamically configured.

Step 4. Analyse and alter frame ontology-this step defines the ontology mechanism based on class structures of associated frames. The class hierarchies of the frame ontology should be thoroughly analysed to track and resolve incompatibilities with current BI-services.

Step 5. Apply outright changes-the outright changes may refer to the overall modifications of the current IoT knowledge base system without affecting its constraints and architectures to achieve the next-level IoT knowledge base system.

2.1. Semantic Knowledge Analytics. In the current business environment, context-aware IoT knowledge analytics produce substantial quantities of daily inputs that influence human thought processes in business and risk handling, at business events, and so forth to ensure the ease of understanding, use, and implementation of BI-services. Knowledge context analysis is not new, but the analytics of the IoT environment create substantially more smart business opportunities for current BI-services, ranging from manufacturing assistance services to transportation and logistics services in numerous BI-applications. Here, we may develop a prospective view of knowledge context analytic scenarios by analysing various knowledge components in accordance with standard BI-services. The semantics focus on the relations between signifiers, such as words, phases, signs, and symbols that are used in a standard knowledge base to assist in BIservices [11, 12]. We may also build a renovated semantic net to meet the requirements of current BI-services for the critical business environment. To study the configurations of the semantic net in a business IoT environment, the business cases, problems, and tactical solutions are considered. The frame-based expert system can also provide standard BIservices. To establish possible frame-based expert systems in the IoT environment, the aggregation, generalisation, and specialisation of BI-objects are considered. The frame ontology provides a mechanism for semantic knowledge discovery and analytics to transform the knowledge of BIproblems into real assistive solutions [13].

The frequent fluctuation of knowledge context scenarios leads to the massive alteration of semantic nets and frames per the BI-service requirements, resulting in several limitations and challenges and often degrading the performance of BI-services. Thus, in the subsequent discussion, we mainly highlight the ontology mechanism through context- and rule-based analytics to provide an effective BI-service in the IoT regulated business environment. The context and rule ontologies have a wider and prospective implementation in upcoming BI-intensive research and development in IoT computing. The European Union has also identified such a vision as a thrust for research in the years leading to 2020 [14].

2.1.1. Context Ontology Outline. The outline defines the overall specifications of BI-scenarios in a standard conceptualised form. Context-aware IoT knowledge analytics require an ontology-based framework to configure semantic knowledge discovery and analytic scenarios for real-time BI-services [15-17]. The context ontology is a typical form of a declarative morphology to document the IoT knowledge base in various customised specification layouts.

2.1.2. Rule Ontology Outline. The rules refer to the activities of standard BI that can be set and configured through a neurofuzzy expert system. Normally, in the standard BI context, the rules constitute the consequences of conditions and actions through a set of linguistic variables and values $[18,19]$. The neurofuzzy features are successfully integrated towards implementing the fuzzy associated business rules over a cognitive neural network environment [20].

2.1.3. Case Analytics. In this example, we consider a business context and rule set for a specific business case. Based on the current ontology, we derive a rule set, and based on the nextlevel ontology, we refine the rule set. This refinement process 
is continued until a substantially more precise inference is achieved to meet the current requirements of the business context. Here, each business context may be treated as a BIservice for the specific business monitoring application. The next-level ontology can be built based on data collected by an IoT evolutionary network connected across the distributed business environment. For a sample case, we execute a series of steps for the possible inference of a new decision.

Step 1. Find a business context associated with a BI-service.

Step 2. Derive a probabilistic fuzzy rule set through possible inferences based on the mechanism of the current ontology.

Step 3. Acquire new contexts from the recent IoT-generated data based on the mechanism of the next-level ontology.

Step 4. Based on new next-level contexts, refine the probabilistic fuzzy rule set.

Step 5. Repeat Step 4 until a maximum precise inference with a higher probabilistic value is obtained.

Step 6. Deduce the result as an effective inference for the stated business intelligence context.

2.1.4. Crossbreed Ontology for IoT Knowledge Base. To fulfil the vision of research and development in BI-intensive IoT computing for the IoT knowledge base system, a crossbreed ontology mechanism that inherits both the features of the current and next-level context ontology and the rule ontology may be suggested. The crossbreed ontology dynamically checks the operations of the IoT knowledge base system as per the current BI requirements and suggests whether to begin reengineering work. The reengineering work includes the prospective modifications and enhancement of contexts and rules that exist in the current operational knowledge base system.

The crossbreed ontology requires a cognitive learning system to track and trace the following BI-service requirements.

(i) Track and trace when an IoT knowledge base reaches a minimum requirement threshold.

(ii) Track and trace what type of reengineering should be conducted on the contexts and rules associated with the BI-services.

(iii) Track and trace the identified contexts and rules that are obsolete and that should be removed from the current knowledge base system.

(iv) Track and trace the analysis of incoming IoT data streams to extract the selective rules and contexts that are to be added to the current knowledge base system.

(v) Track and trace what knowledge can be used for a specific business context scenario.

(vi) Track and trace when to use the knowledge for a specific business context scenario.

(vii) Track and trace how to use the knowledge for a specific business context scenario.
To implement a crossbreed ontology for an IoT knowledge base, we introduce two types of knowledge analytics that are involved in the IKR framework-reverse knowledge analytics and forward knowledge analytics. Here, we design two algorithms that use both contexts and rules as the sources of operations for BI-intensive IoT computing research and development for the IoT knowledge base system.

Algorithm 1 describes a knowledge reanalytic policy for tracking and tracing BI-service requirements. The knowledge reanalytics follow both forward analytic and reverse analytic strategies. In the reverse analytic strategy, the analytics are applied to the current superseded knowledge base to extract the current context ontologies and rule ontologies, as described in Algorithm 2. Algorithm 3 describes a forward analytic strategy, in which the analytics are applied to the next-level knowledge base to extract the next-level innovated context ontologies and rule ontologies, and a neurofuzzy implementation mechanism is configured in Algorithm 4. Based on both current and next-level ontologies, the tracking and tracing of BI-service requirements can be effectively performed through a comprehensive IoT environment in which things (man, machines, places, and processes) are interconnected without any network and communication barriers to meet automated service requirements such as selfdecisions, self-actuations, self-optimisation, self-control, and self-coordination.

Algorithm 1 (knowledge reanalytics for tracking and tracing BI-service requirements).

(1) Input-Accept current and next-level BI context ontologies and rule ontologies;

(2) Process-Implement a neuro-fuzzy associated machine learning algorithm;

(3) Output-Track and trace the BI-service requirements;

(4) knowledge-re-analytic ( ) // initial function

(5) \{

(6) reverse-knowledge-analytic ( ) // current ontologies

(7) forward-knowledge-analytic ( ) // next-level ontologies

(8) $\}$

(9) find-service-request ( )

(10) \{

(11) get the auto-execute service requests;

(12) $\}$

(13) execute-service-request ( )

(14) \{

(15) get crossbreed ontology;

(16) neuro-fuzzy-analytic ( );

(17) // trace requests to a pre-configured neural network embedded with trained fuzzy rule ontologies and context ontologies;

(18) \} 
(19) track-trace-service ( )

(20) \{

(21) post track and trace BI-service requirements;

(22) $\}$

(23) IF(track-trace-service $=$ false $) / /$ complete unacceptable

(24) \{

(25) goto Step (16);

(26) \}

(27) else

(28) \{

(29) wait for next scheduled routine;

(30) goto Step (4);

(31) $\}$

Algorithm 2 (reverse knowledge analytic strategy).

(1) Input-Current knowledge base;

(2) Process-Analysis of current knowledge contexts and rules to meet the BI-service requirements;

(3) Output-Current knowledge contexts and rules;

(4) reverse-knowledge-analytic ( )

(5) \{

(6) while (analysis is not over)

(7) \{

(8) accept BI-service requirement;

(9) find the knowledge context from current knowledge base;

(10) track the suitable rule set per knowledge context;

(11) $\}$

(12) return (knowledge context and rule set);

(13) $\}$

Algorithm 3 (forward knowledge analytic strategy).

(1) Input-Next-level IoT database

(2) Process-Analysis of next-level knowledge contexts and rules to meet the BI-service requirements

(3) Output-Next-level knowledge contexts and rules

(4) forward-knowledge-analytic ( )

(5) \{

(6) accept IoT data streams;

(7) find the contexts;

(8) find linguistic variable and values;

(9) set fuzzy membership grades;

(10) generate rule sets based on contexts;

(11) use probabilistic method to generate possible inferences on rule sets;
(12) $\}$

(13) return (next-level contexts and rules);

(14) $\}$

Algorithm 4 (neurofuzzy implementation).

(1) Input-BI-contexts;

(2) Process-Neuro-fuzzy analytic operation;

(3) Output-Probabilistic inference;

(4) neuro-fuzzy-analytic ( )

(5) $\{\{$

(6) set current and next-level BI-contexts as input;

(7) pass through a fuzzification process;

(8) pass through current rule ontology for possible inferences;

(9) pass through next-level rule ontology for probabilistic refinements;

(10) pass through a normalisation process to normalise rule strength;

(11) pass through a defuzzification process;

(12) pass through a summation process with a BIrequirement threshold;

(13) $\}$

(14) return the most precise inference with the highest probabilistic value.

(15) $\}$.

\section{Analysis and Discussion}

Here, we analyse and discuss the implementation and operation of the IKR framework in an IoT knowledge base for prospective BI-intensive services through statistical and computational analysis approaches. The effective functional and operational analysis of the IKR framework improves the quality of BI-services, and the quality of BI-services is measured as the precision of inference with the highest probabilistic value. Prior to the analysis, we review some works that are associated with knowledge engineering and reengineering systems, frameworks, tools, and others that previously been applied to different BI-services in the IoT environment. In Table 1, a review list of certain distinct BIservice applications associated with various proposed technologies in the IoT environment is presented, and almost all service applications require the semantic knowledge analytics from large-scale IoT data that are distributed across the business environment.

\subsection{Knowledge Analytics and Visualisation}

3.1.1. Case Analytics. In case of analytics, we use an analysis and visualisation of BI-service applications. In a typical business organisation, the standard BI-services are the probabilistic estimation of customer buying attitudes, item 
TABLE 1: List of certain distinct BI-service applications associated with proposed technologies in the IoT environment.

\begin{tabular}{lll}
\hline Name of works & Name of BI-service apps & Proposed technologies \\
\hline Bi et al. [25] & Modern manufacturing service & IoT and cloud computing \\
Grigori et al. [26] & E-commerce service applications & Business process monitoring systems \\
Jara et al. [27] & IoT-based medical service application & ICT-IoT framework \\
Panigrahi et al. [28] & Credit card fraud detection service & D-S theory and Bayesian learning system \\
Kiritsis [29] & Product lifecycle management service & Closed-loop PLM framework \\
Qu et al. [30] & Transport logistic service & Ontology framework \\
Csikósová and Antošová [31] & Supply chain management service & Smart logistic system \\
\hline
\end{tabular}

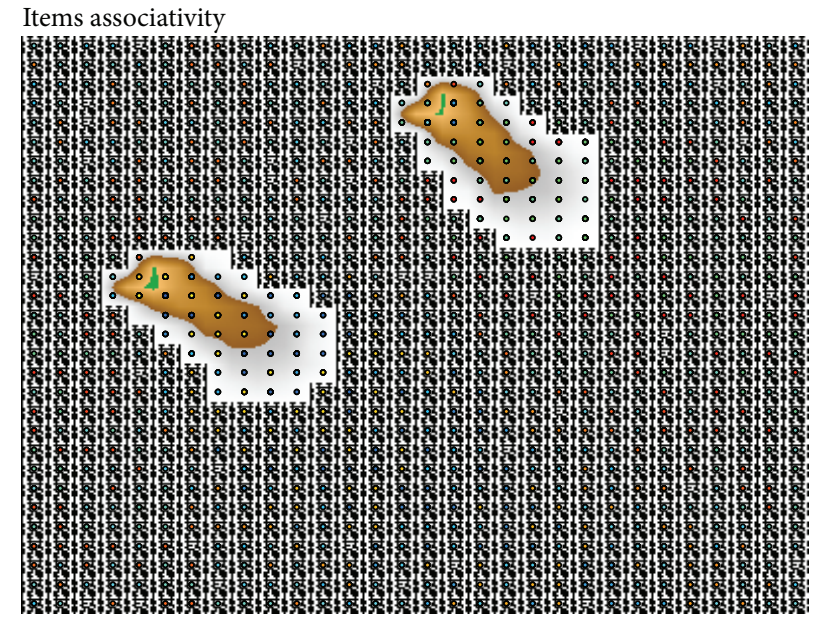

0.012 0.876

FIGURE 4: Item associativity based on customer trade transactions.

associativity based on transactions, service reliability, trading trend sustainability, and trade strategy.

Figure 4 describes the anticipated item associativity based on the transactions performed by customers. The buying behaviour of a customer can be analysed through a series of transactions with that customer. The probable item associativity grid is a self-organising map that is configured through 100 training rounds with an adaption radius of 10 . These knowledge analytics and visualisation map suggest how trade organisations can increase their items' associativities based on customer transactions. In the above item associativity scenario, two white-shaded areas are identified; the upper whiteshaded area contains a higher associativity of homogeneous items, whereas the lower white-shaded area contains a higher associativity of heterogeneous trade items. Such associativity analysis is prepared based on the transaction data of the customers at different instances.

Service reliability is an important BI-service that should be measured based on customer buying behaviours and attitudes that support further estimation of the trading trend sustainability of the organisation. Additionally, based on the probabilistic estimations of customer buying attitudes, the item associativity, service reliability, trading trend sustainability, and overall trade strategy of a business organisation can be well estimated.
For the above BI-services, individual BI-ontologies can be built to provide specified BI-services. The IoT environment can be effectively used to implement parallel and distributed data computing and analytics across a geographically distributed business environment to provide widespread BIservices.

(i) A customer buying attitude ontology estimates customer behaviours and buying attitudes based on trade transactions.

(ii) An item associativity ontology estimates a probable item associativity based on customer buying attitudes and on the transactions of a business organisation.

(iii) A service reliability ontology estimates the reliability of business processes and products based on a sequence of customer behaviours and buying attitudes.

(iv) A trend sustainability ontology estimates the probable sustainability of the product and services of a business organisation.

(v) A trade strategy ontology forecasts the trade strategy of a business organisation for a specific period of time based on the analytics of the previous ontologies.

3.2. Computational Analysis. In this computational analysis, we explore a neural representation and multilayer neurofuzzy representation of BI-rules and contexts for probabilistic knowledge inferences. The neurofuzzy rule-based system integrates the good policies of the standalone neural system, fuzzy system, and rule-based system to increase performance in terms of the knowledge representation, tolerance, learning and discovery ability, enhanciation ability, and reusability. The main objective of the integrated system is to provide the BI-service in the TQM (total quality management) standard. Figure 5 describes a neural representation of BI-rules and a context for probabilistic knowledge inferences. Here, the current BI-rules along with the next-level BI-rules are used in the BI-contexts for the possible knowledge inferences such that each inference is characterised by a probabilistic value. The higher the probabilistic value associated with an inference, the greater its chances of occurring with respect to that knowledge context.

Figure 6 describes a neurofuzzy representation of BIrules and contexts for probabilistic knowledge inferences in which the fuzzification layer uses fuzzy sets in the antecedents 


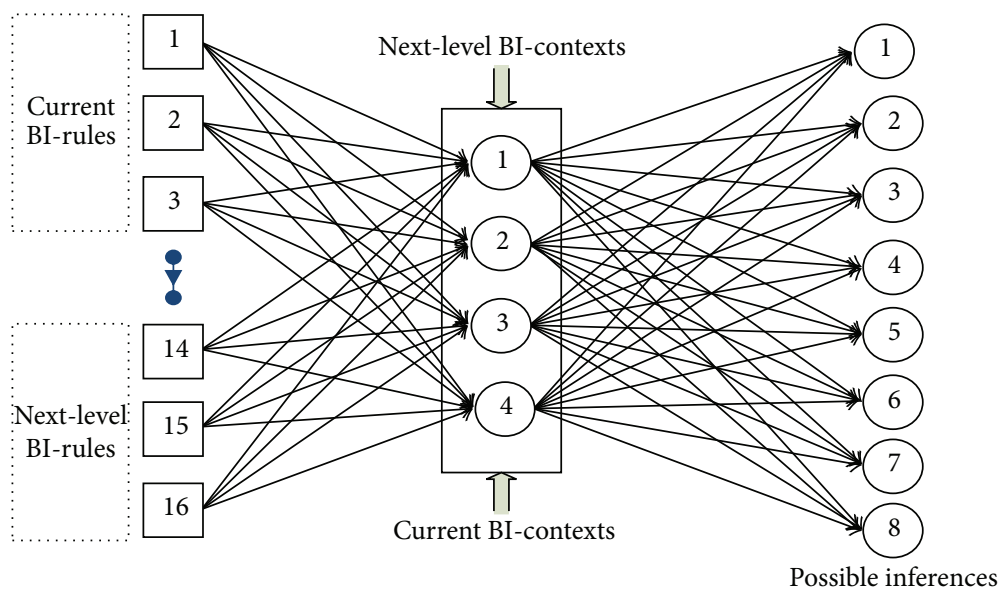

FIGURE 5: Neural network representation of BI-rules and contexts for probabilistic knowledge inference.

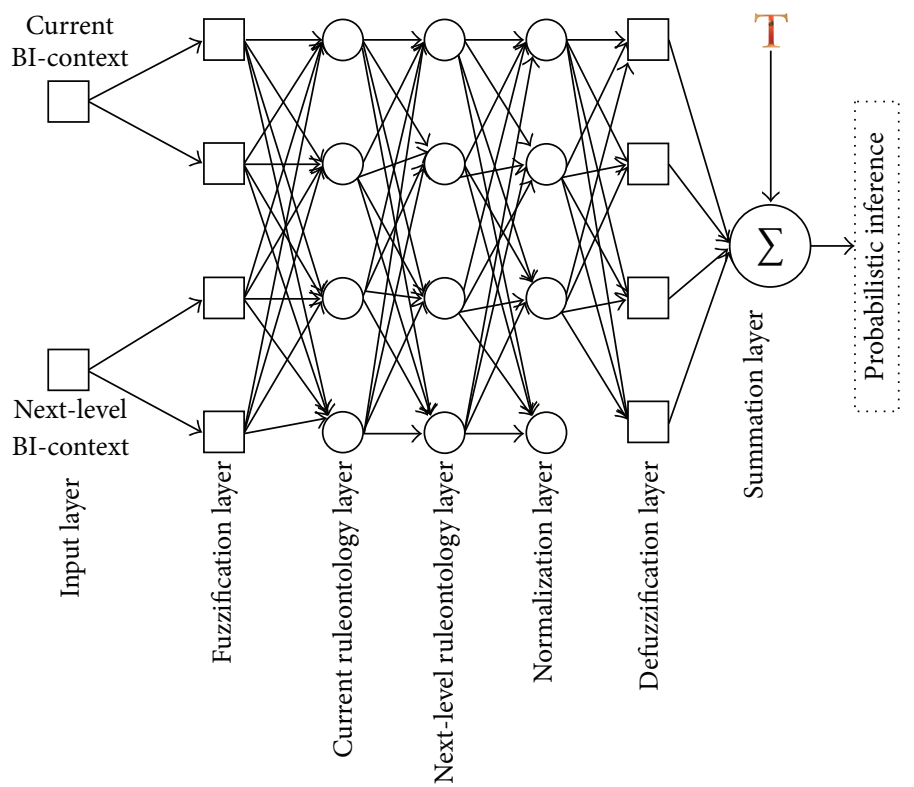

FIGURE 6: Neurofuzzy representation of BI-rules and contexts for probabilistic knowledge inference.

of the fuzzy rules. In real-time BI scenarios, we consider each fuzzy set to be linked to every individual BI-rule. The current and next-level rule ontology layers are responsible for handling the respective rules to refine the BI-context to generate knowledge inferences with higher probabilistic value.

The defuzzification layer helps achieve a crisp output from integrated fuzzy sets based on the BI-rules and contexts. The current and next-level BI-contexts are fetched to a multilayer neurofuzzy system that maps all current and next-level rule ontologies to produce the probabilistic knowledge inferences.

To normalise the firing strength of the current and nextlevel BI-rules, a normalisation layer is used. To obtain the higher probabilistic value associated with inferences, BIservice requirement threshold ( $\mathrm{T}$ ) must be set by the domain expert in the summation layer. For our implementation, we set the target threshold (T) as greater than zero to filter all positive inference values because negative inference values do not make sense in the standard defuzzification range of BI-service instances. The overall architecture in Figure 6 is described as follows. At a given time, we consider two knowledge contexts, one from the current knowledge base and another from the next-level knowledge base. From each knowledge context, two fuzzy sets are derived. Those four sets refer to all four rules of the current knowledge base and further extend the reference to the rules of the nextlevel knowledge base to refine and finalise the knowledge inferences associated with the probabilistic values. The configuration of a neurofuzzy system entirely depends on the BIservice requirements, the business domain, and the serviceoriented architecture provided by the concerned business organisation.

We now discuss the learning mechanism of the preconfigured neurofuzzy system that plans the BI-functions 


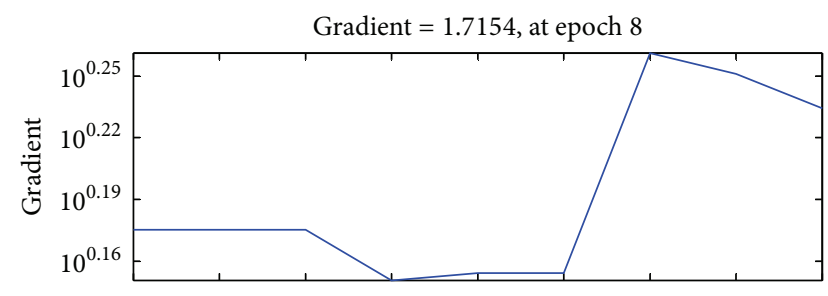

FIGURE 7: Gradient analysis during the learning phase (the $x$-axis is the epoch, and the $y$-axis is the gradient).

and maps the possible functions onto the neural network platform to minimise the prediction error based on the targets [21, 22]. Once the system is embedded with the smart inference framework, an appropriate learning process should be initiated to test the performance level. The gradient-based algorithm is an optimised algorithm in which a weight matrix can be successfully updated during the training phase of the system [23]. We map the BI-functional activity instances onto the neurofuzzy environment, which learns with the gradientbased algorithm associated with the functional estimations of the BI-services. We use a conjugate gradient as the learning algorithm for the input $\mathrm{BI}$ instances and find that the gradient value $=1.7154$ at epoch 8 during the learning phase (see Figure 7). During the learning phase, the gradient initially suddenly decreases and increases at the fifth epoch. The BI instances are randomly allocated to five samples such that $70 \%$ of the instances are used for training, $15 \%$ of the instances are used for validation, and the remaining $15 \%$ are used for testing. In this analysis, we achieve zero error or a minimum error $e=-0.00742$ among the maximum number of BI instances, as depicted in Figure 8. The zero error or very low error indicates the better measured performance of the learning system. As soon as the error computation is accomplished, the mean square error can be computed to determine the average square difference between the computed output and the target output to analyse the overall performance.

The following four BI-service contexts are taken as inputs to the neurofuzzy system:

(i) Input 1: customer buying attitude (CBA),

(ii) Input 2: item associativity (IA),

(iii) Input 3: service reliability (SR),

(iv) Input 4: trend sustainability (TS).

The respective ontologies are used to determine the probabilistic values of the four BI-service contexts as inputs. The following is the output of the neurofuzzy system:

(i) Output 1: overall trade strategy (OTS).

Various real-time data sets are analysed to visualise the probabilistic values of the above inputs in a BI-service context and to normalise and transform those data sets into a standard fuzzy relational database structure within the standard fuzzy range $[0,1]$.

Table 2 is a statistical survey analytic report used to quantify the service parameters based on a customer's pretrading views, posttrading views, and transactions along

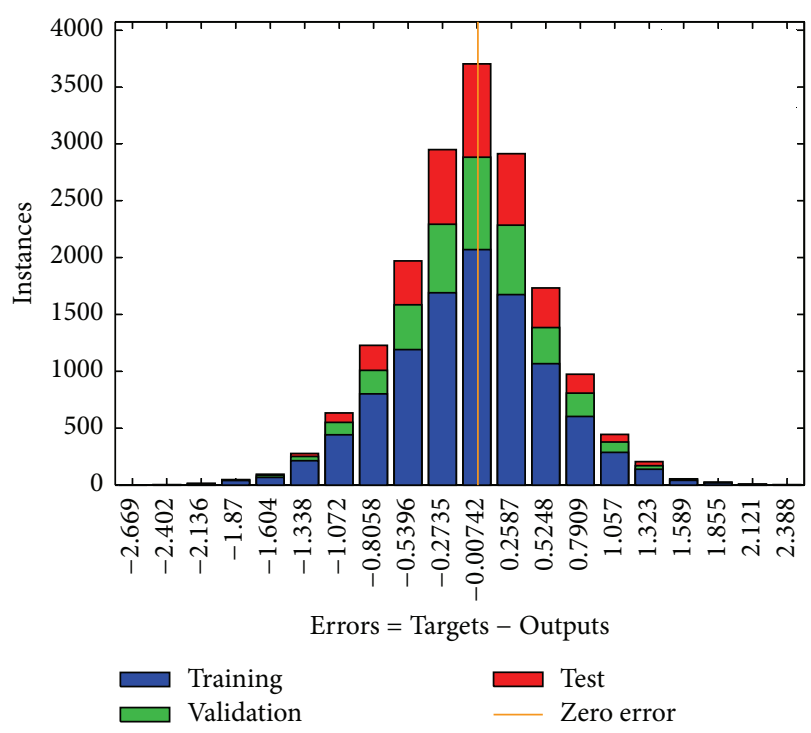

FIGURE 8: Error analysis during learning phase.

TABLE 2: Statistical survey analysis of BI-service contexts in standard fuzzy range.

\begin{tabular}{lcccc}
\hline Parameter (data type) & Min & Max & Average & Deviation \\
\hline Buying attitude (real) & 0.050 & 0.905 & 0.142 & 0.171 \\
Item associativity (real) & 0.012 & 0.876 & 0.396 & 0.259 \\
Service reliability (real) & 0.000 & 0.988 & 0.364 & 0.227 \\
Trend sustainability (real) & 0.068 & 1.000 & 0.576 & 0.284 \\
\hline
\end{tabular}

with the prospective of item arrangements. Those views are normalised to a standard fuzzification range and analysed to design the statistical analysis report. This statistical analysis report of BI-services is implemented in a neurofuzzy environment to enable the possible prediction of knowledge inferences. A detailed statistical analysis of these data sets is described in Table 2 for further analysis and exploration.

Three fuzzy membership grades are used to quantify the four input parameters that function as BI-service contexts. For our implementation, we use three fuzzy membership grades to qualify the inputs as poor, average, or good BIservice contexts in the standard fuzzified range.

(i) $\mu_{(A)}{ }^{(x)}: x \in X$ and $X \rightarrow[0.0,0.4]$, if $x$ is a poor BIservice context and $A$ is the subset of $X$.

(ii) $\mu_{(A)}{ }^{(x)}: x \in X$ and $X \rightarrow[0.6,1.0]$, if $x$ is a good BIservice context and $A$ is the subset of $X$.

(iii) $\mu_{(A)}^{(x)}: x \in X$ and $X \rightarrow[0.4,0.7]$, if $x$ is an average BI-service context and $A$ is the subset of $X$.

We analyse eight current-level BI-rules for the sake of implementation in a neurofuzzy environment. The nextlevel BI-context and rules are traced as the probability of occurrences and will be considered based on the newly changing business scenarios. In the BI-rules, we use the same linguistic variables, poor, good, and average, along with their 

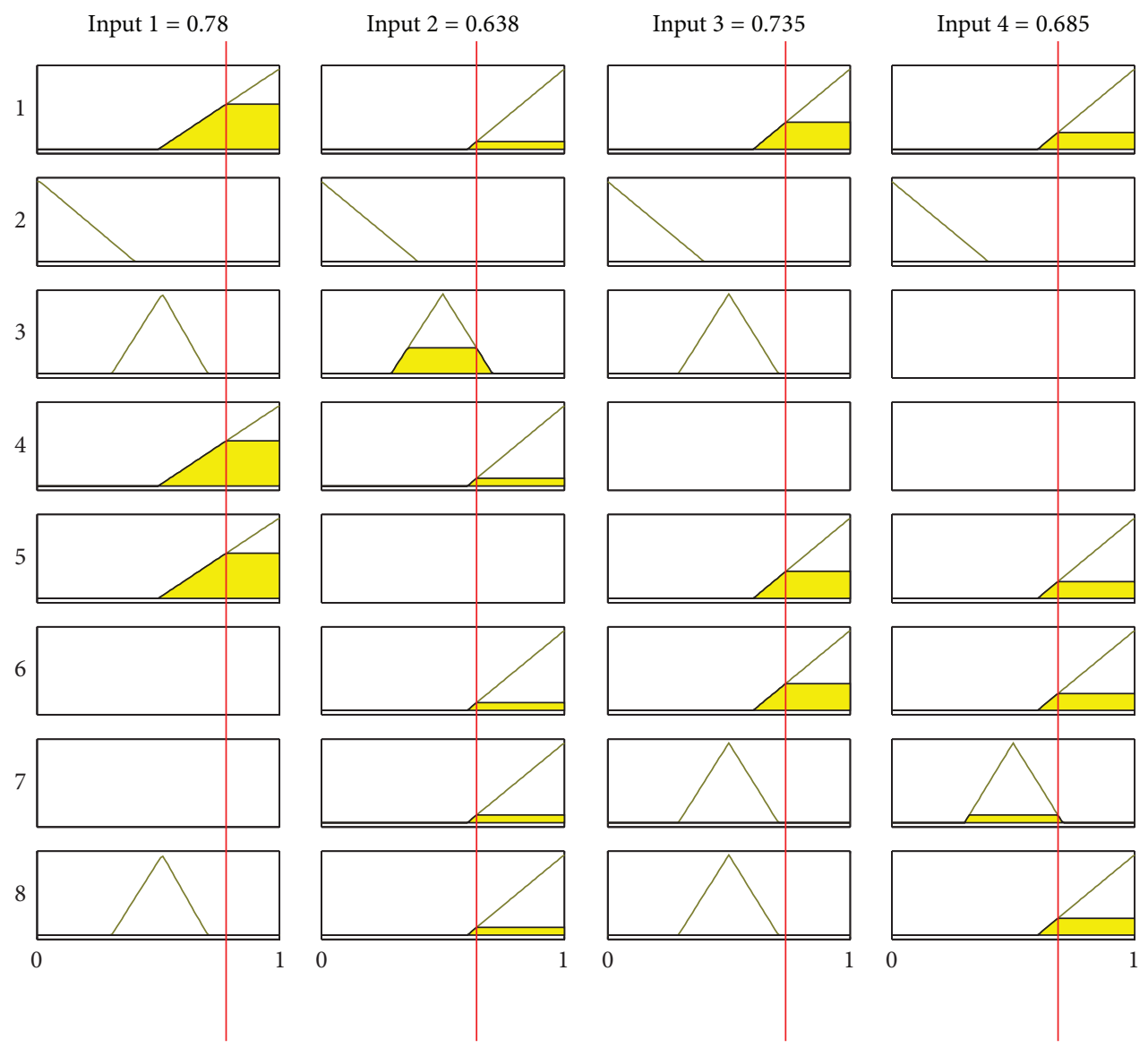

Output $1=0.725$
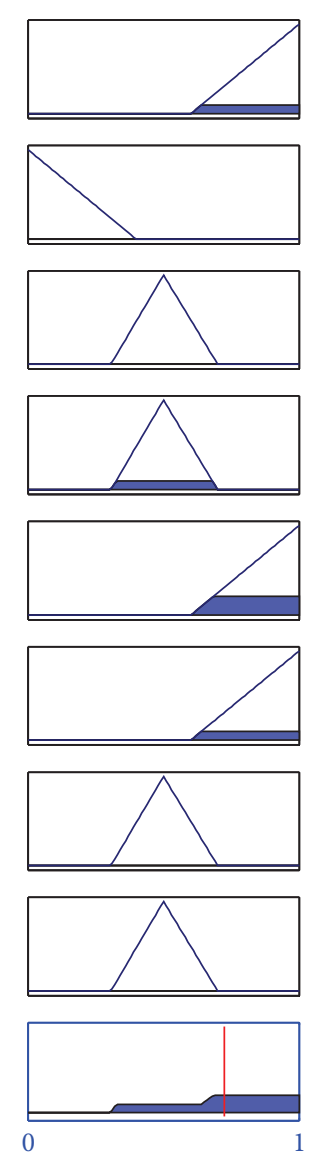

Figure 9: Neurofuzzy implementations of BI-rules and contexts.

predefined membership grades. We use a business case to derive the following eight rules.

\section{Business Rule Analytics}

Rule 1. If $(\mathrm{CBA}=\operatorname{good})$ and $(\mathrm{IA}=\operatorname{good})$ and $(\mathrm{SR}=\operatorname{good})$ and $(\mathrm{TS}=$ good $)$ then $(\mathrm{OTS}=$ good $)$.

Rule 2. If $(\mathrm{CBA}=$ poor $)$ and $(\mathrm{IA}=$ poor $)$ and $(\mathrm{SR}=$ poor $)$ and (TS $=$ poor $)$ then (OTS $=$ poor).

Rule 3. If $(\mathrm{CBA}=$ average $)$ and $(\mathrm{IA}=$ average $)$ and $(\mathrm{SR}=$ average $)$ and (TS $=$ average $)$ then (OTS $=$ average).

Rule 4. If $(\mathrm{CBA}=\operatorname{good})$ and $(\mathrm{IA}=\operatorname{good})$ then $(\mathrm{OTS}=$ average).

Rule 5. If $(\mathrm{CBA}=\operatorname{good})$ and $(\mathrm{SR}=\operatorname{good})$ and $(\mathrm{TS}=\operatorname{good})$ then $(\mathrm{OTS}=$ good $)$.

Rule 6. If $(\mathrm{IA}=\operatorname{good})$ and $(\mathrm{SR}=\operatorname{good})$ and $(\mathrm{TS}=\operatorname{good})$ then $(\mathrm{OTS}=$ good $)$.

Rule 7. If $(\mathrm{IA}=$ average $)$ and $(\mathrm{SR}=\operatorname{good})$ and $(\mathrm{TS}=\operatorname{good})$ then $(\mathrm{OTS}=$ good $)$.
Rule 8. If $(\mathrm{CBA}=\operatorname{good})$ and $(\mathrm{IA}=\operatorname{good})$ and $(\mathrm{SR}=\operatorname{good})$ and $(\mathrm{TS}=$ poor $)$ then (OTS $=$ average).

We implement eight rules, of which the first three (Rules 1 to 3 ) are the universal rules, which may not change with the changing business scenarios. However, the last five rules (Rules 4 to 8 ) dynamically change with the changing business contexts and scenarios. Based on the new analytic operation associated with the next-level contexts, the next-level rules must be refined accordingly. Additionally, based on the nextlevel analytic scenarios, the IoT knowledge base should be dynamically reengineered to meet the future BI-service requirements. The next-level BI-context and rules are traced as probabilities of occurrence and will be considered based on the newly changing business scenarios.

Figure 9 describes the neurofuzzy implementations of BI-contexts and rules. The four inputs are the four BIcontexts described earlier. Here, eight possible rules that use the probabilistic values of those four inputs to produce the desired output value, that is, OTS value, are generated. The neurofuzzy system environment exercises substantially more real-time control to regulate numerous sensitive applications [24]. Thus, we may configure a neurofuzzy environment to simulate different neural network configurations through the 
fuzzified data sets relating to the BI-functional activities for numerous emergent BI applications.

\section{Conclusions and Future Work}

In our work, we discuss an IKR framework for reengineering a superseded IoT knowledge base into a renovated knowledge base system that can ensure higher operational efficiency compared to the previous superseded knowledge base system as a result of the in-time reengineering of the useful knowledge contexts. The operations related to IoT knowledge analytics are discussed along with possible explorations of semantics, frames, rules, and ontologies. The reengineering process is certainly more cost effective than new engineering, especially when renovating an IoT knowledge base system towards modernising business operations to provide BIservices.

However, there are various areas that need to be explored in BI-service applications. In BI-applications, the time to gain insight is long, and the cost of the insight is high. Thus, business executives start to consider new IoT regulated BItechnologies to modernise their business environment with self-regulated operations.

We further focus on implementing new deep learning tools that can be successfully applied in semantic knowledge analytics for numerous BI-applications.

\section{Conflict of Interests}

The authors declare that there is no conflict of interests regarding the publication of this paper.

\section{Acknowledgments}

The financial support provided by the Ministry of Science and Technology, Taiwan, through Grants MOST 103-2221-E182-053, MOST 103-2511-S-182-003, and CMRPD3E0061 of Chang Gung University is gratefully acknowledged.

\section{References}

[1] R. Khan, S. U. Khan, and R. Zaheer, "Future internet: the internet of things architecture, possible applications and key challenges," in Proceedings of the 10th International Conference on Frontiers of Information Technology (FIT'12), pp. 257-260, December 2012.

[2] H. Al-Aqrabi, L. Liu, R. Hill, and N. Antonopoulos, "Cloud BI: future of business intelligence in the cloud," Journal of Computer and System Sciences, vol. 81, no. 1, pp. 85-96, 2015.

[3] T. Knabke and S. Olbrich, "Understanding Information System agility-the example of business intelligence," in Proceedings of the 46th Annual Hawaii International Conference on System Sciences (HICSS '13), pp. 3817-3826, IEEE, January 2013.

[4] R. Adderley, P. Seidler, A. Badii, M. Tiemann, F. Neri, and M. Raffaelli, "Semantic mining and analysis of heterogeneous data for novel intelligence insights," in Proceedings of the 4th International Conference on Advances in Information Mining and Management (IMMM '14), pp. 36-40, Paris, France, July 2014.
[5] B. Gupta and U. Raja, "Teaching analytics, decision support, and business intelligence: challenges and trends," in Reshaping Society through Analytics, Collaboration, and Decision Support, vol. 18 of Annals of Information Systems, pp. 205-209, Springer, 2015.

[6] M. Fuchs, W. Höpken, and M. Lexhagen, "Applying business intelligence for knowledge generation in tourism destinationsa case study from Sweden," in Tourism and Leisure, pp. 161-174, Springer Fachmedien Wiesbaden, Wiesbaden, Germany, 2015.

[7] M. Hwang, J. Kim, J. Gim, S. K. Song, H. Jung, and D. H. Jeong, "Domain terminology collection for semantic interpretation of sensor network data," International Journal of Distributed Sensor Networks, vol. 2014, Article ID 827319, 9 pages, 2014.

[8] Q. Wu, G. Ding, Y. Xu et al., "Cognitive internet of things: a new paradigm beyond connection," IEEE Internet of Things Journal, vol. 1, no. 2, pp. 129-143, 2014.

[9] N. Mishra, H. Chang, and C. Lin, "Data-centric knowledge discovery strategy for a safety-critical sensor application," International Journal of Antennas and Propagation, vol. 2014, Article ID 172186, 11 pages, 2014.

[10] N. Mishra, C.-C. Lin, and H.-T. Chang, "Cognitive inference device for activity supervision in the elderly," The Scientific World Journal, vol. 2014, Article ID 125618, 12 pages, 2014.

[11] A. Hussain, K. Latif, A. T. Rextin, A. Hayat, and M. Alam, "Scalable visualization of semantic nets using power-law graphs," Applied Mathematics and Information Sciences, vol. 8, no. 1, pp. 355-367, 2014.

[12] S. Martins, D. Rodríguez, and R. García-Martínez, "Deriving processes of information mining based on semantic nets and frames," in Modern Advances in Applied Intelligence, vol. 8482 of Lecture Notes in Computer Science, pp. 150-159, Springer, 2014.

[13] M. Negnevitsky, Artificial Intelligence: A Guide to Intelligent Systems, 3rd edition, 2011.

[14] O. Vermesan, P. Friess, P. Guillemin et al., "Internet of things strategic research roadmap," in Internet of Things-Global Technological and Societal Trends, pp. 9-52, River Publishers, 2011.

[15] M. Sohn, S. Jeong, and H. J. Lee, "Case-based context ontology construction using fuzzy set theory for personalized service in a smart home environment," Soft Computing, vol. 18, no. 9, pp. 1715-1728, 2014.

[16] A. K. Dey, "Understanding and using context," Personal and Ubiquitous Computing, vol. 5, no. 1, pp. 4-7, 2001.

[17] S. N. A. U. Nambi, C. Sarkar, R. V. Prasad, and A. Rahim, "A unified semantic knowledge base for IoT", in Proceedings of the IEEE World Forum on Internet of Things (WF-IoT'14), pp. 575580, March 2014.

[18] W. Wang, S. De, R. Toenjes, E. Reetz, and K. Moessner, "A comprehensive ontology for knowledge representation in the internet of things," in Proceedings of the 11th IEEE International Conference on Trust, Security and Privacy in Computing and Communications (TrustCom '12), pp. 1793-1798, June 2012.

[19] C. Perera, A. Zaslavsky, P. Christen, and D. Georgakopoulos, "Context aware computing for the internet of things: a survey," IEEE Communications Surveys \& Tutorials, vol. 16, no. 1, pp. 414-454, 2014.

[20] H. Zhou, Y. Wang, and K. Cao, "Fuzzy D-S theory based fuzzy ontology context modeling and similarity based reasoning," in Proceedings of the 9th International Conference on Computational Intelligence and Security (CIS '13), pp. 707-711, IEEE, December 2013. 
[21] J.-S. R. Jang, "ANFIS: adaptive-network-based fuzzy inference system," IEEE Transactions on Systems, Man and Cybernetics, vol. 23, no. 3, pp. 665-685, 1993.

[22] A. Lemos, W. Caminhas, and F. Gomide, "Multivariable gaussian evolving fuzzy modeling system," IEEE Transactions on Fuzzy Systems, vol. 19, no. 1, pp. 91-104, 2011.

[23] A. M. Silva, W. Caminhas, A. Lemos, and F. Gomide, "A fast learning algorithm for evolving neo-fuzzy neuron," Applied Soft Computing Journal, vol. 14, pp. 194-209, 2014.

[24] S. K. Routray, N. Nayak, and P. K. Rout, "Design of a nonlinear fuzzy controller based on differential evolution for UPFC control," Journal of Bioinformatics and Intelligent Control, vol. 2, no. 4, pp. 305-315, 2013.

[25] Z. Bi, L. D. Xu, and C. Wang, "Internet of things for enterprise systems of modern manufacturing," IEEE Transactions on Industrial Informatics, vol. 10, no. 2, pp. 1537-1546, 2014.

[26] D. Grigori, F. Casati, M. Castellanos, U. Dayal, M. Sayal, and M.C. Shan, "Business process intelligence," Computers in Industry, vol. 53, no. 3, pp. 321-343, 2004.

[27] A. J. Jara, M. A. Zamora, and A. F. G. Skarmeta, "An architecture based on internet of things to support mobility and security in medical environments," in Proceedings of the 7th IEEE Consumer Communications and Networking Conference (CCNC '10), pp. 1-5, IEEE, Las Vegas, Nev, USA, January 2010.

[28] S. Panigrahi, A. Kundu, S. Sural, and A. K. Majumdar, "Credit card fraud detection: a fusion approach using Dempster-Shafer theory and Bayesian learning," Information Fusion, vol. 10, no. 4, pp. 354-363, 2009.

[29] D. Kiritsis, "Closed-loop PLM for intelligent products in the era of the Internet of things," Computer Aided Design, vol. 43, no. 5, pp. 479-501, 2011.

[30] C. Qu, F. Liu, and M. Tao, "Ontologies for the transactions on IoT," International Journal of Distributed Sensor Networks, vol. 2015, Article ID 934541, 12 pages, 2015.

[31] A. Csikósová and M. Antošová, "Supply chain management in condition of production company," in Applied Mechanics and Materials, vol. 718, pp. 168-172, 2015. 


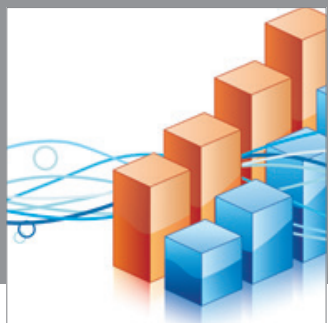

Advances in

Operations Research

mansans

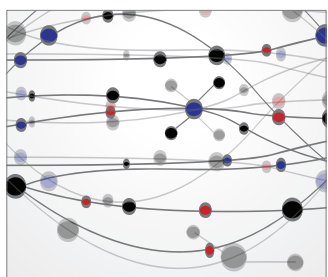

The Scientific World Journal
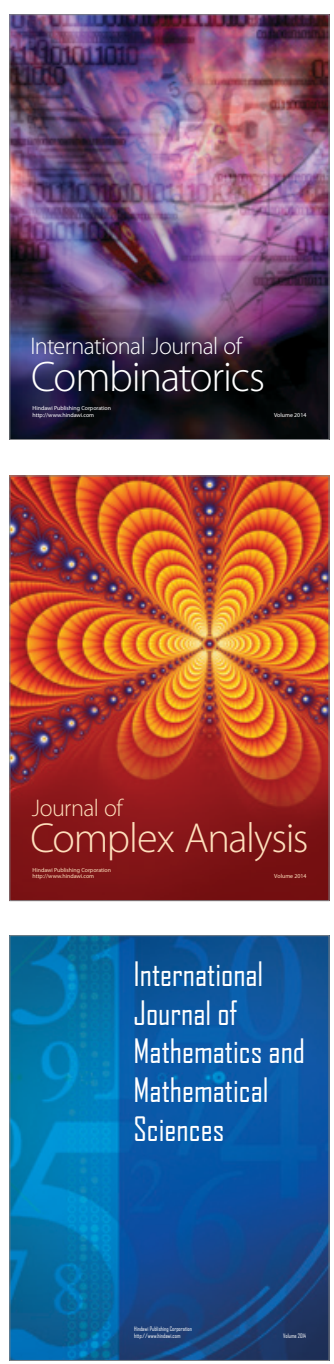
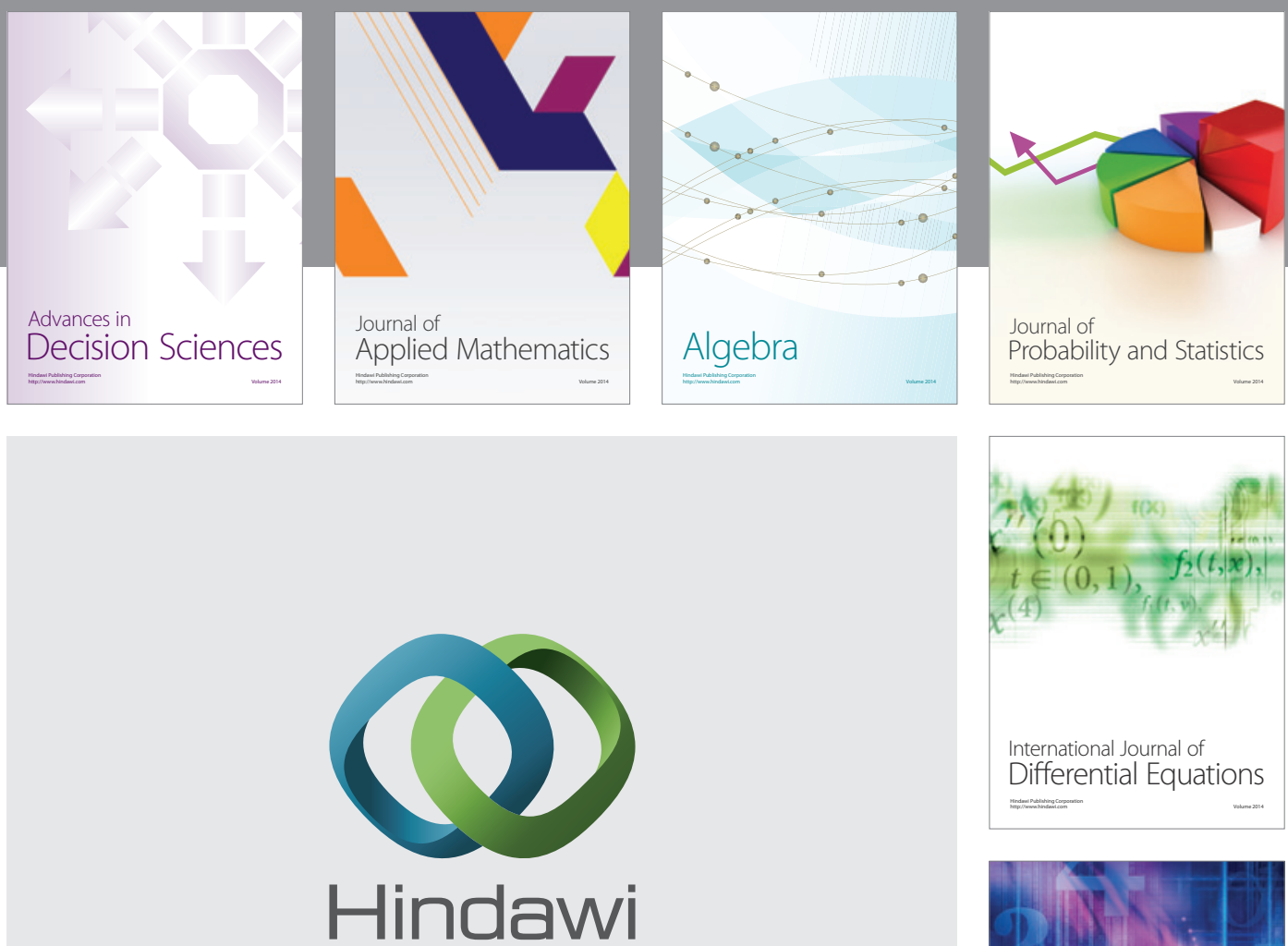

Submit your manuscripts at http://www.hindawi.com
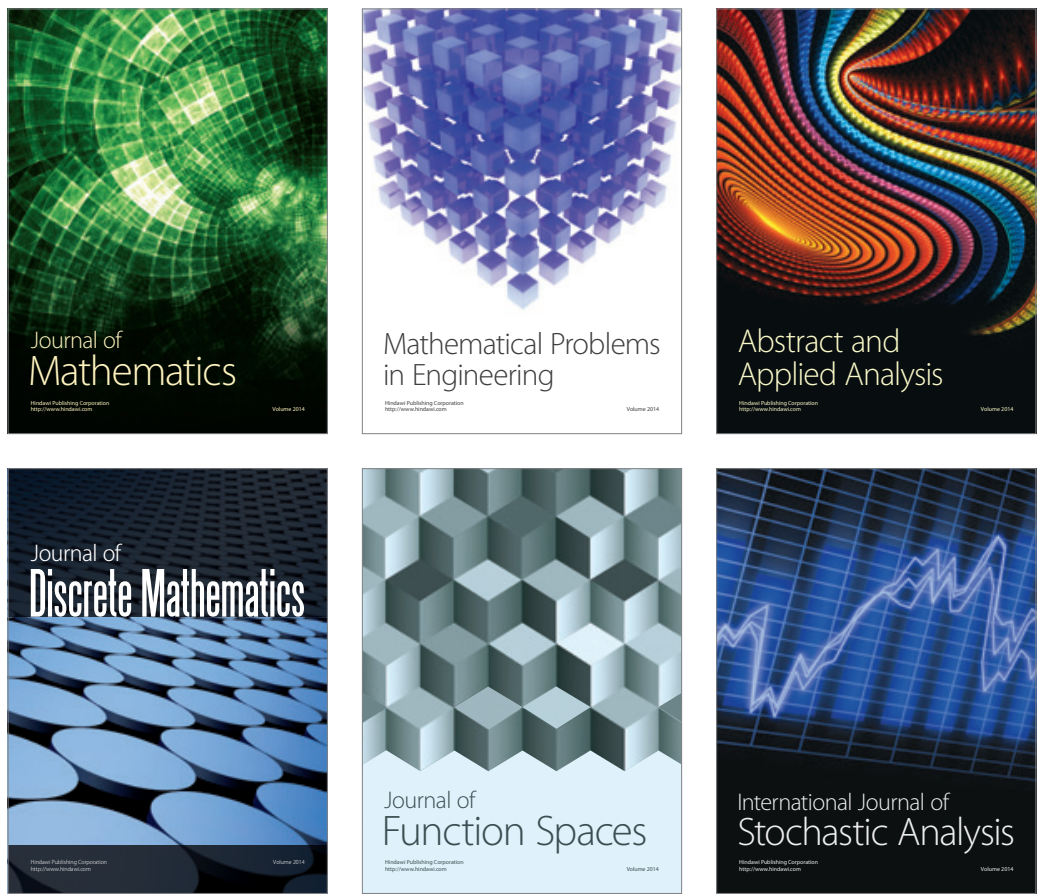

Journal of

Function Spaces

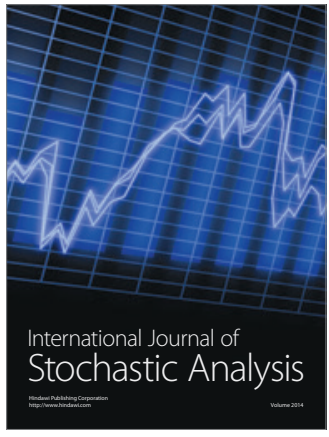

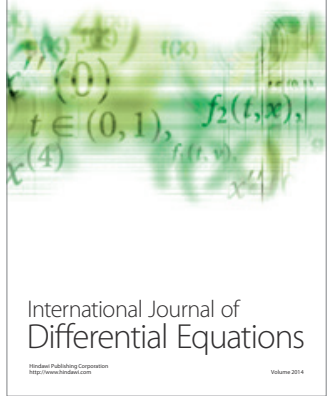
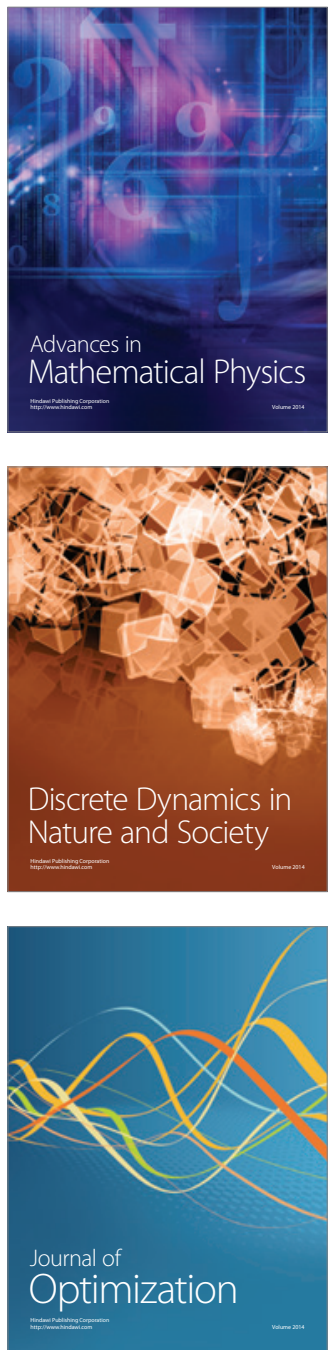